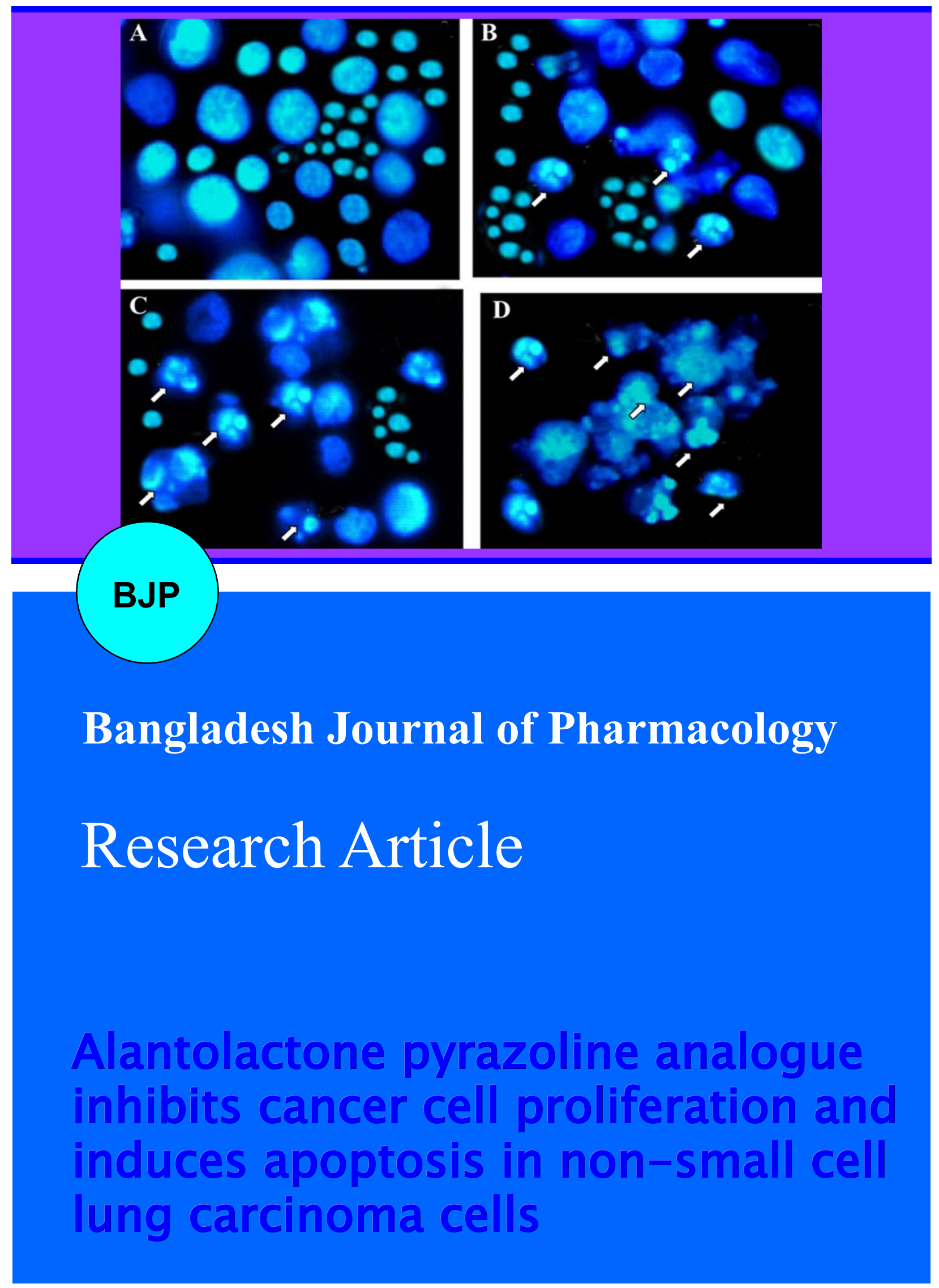




\title{
Alantolactone pyrazoline analogue inhibits cancer cell proliferation and induces apoptosis in non-small cell lung carcinoma cells
}

\author{
Jing lv', Ming-Qin Cao ${ }^{2}$ and Jian-Chun Yus \\ ${ }^{1}$ Department of Oncology, The Affiliated Hospital of Qingdao University, Qingdao 266000, China; ${ }^{2}$ Health \\ Examination Center, Dongying People's Hospital, Dongying 257091, China; ${ }^{3}$ Department of Emergency, Linzi \\ District People's Hospital, Zibo 255400, China.
}

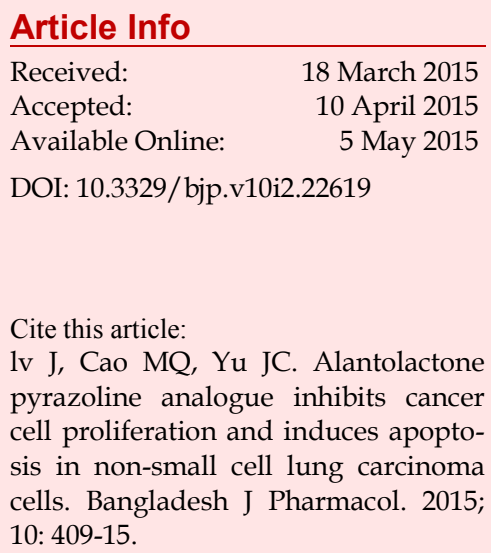

lv J, Cao MQ, Yu JC. Alantolactone pyrazoline analogue inhibits cancer cell proliferation and induces apoptosis in non-small cell lung carcinoma cells. Bangladesh J Pharmacol. 2015; 10: 409-15

Abstract
The aim of the current study was to evaluate the anticancer and apoptotic
effects of alantolactone pyrazoline analogue in human non-small cell lung
cancer (NCI-H460) cells. MTT (3-(4,5-dimethylthiazol-2-yl)-2,5-diphenyltetra-
zolium bromide) assay was used to evaluate the cell viability while as
fluorescence microscopy was used to assess the effect on apoptosis, cellular
and nuclear morphology. Flow cytometry evaluated the effect of APA on cell
cycle arrest in these cells. The results revealed that APA induced potent, time
and dose-dependent cytotoxic effects on the growth of NCI-H460 cells. It also
inhibited colony forming tendency as well as cell invasion capability of these
cancer cells. APA induced dose-dependent nuclear and cellular morphological
effects including chromatin condensation and DNA fragmentation. Flow
cytometry revealed that the anticancer effects of APA might be due to its cell
cycle arrest inducing tendency in G0/G1 phase of the cell cycle.

\section{Introduction}

Lung cancer is still the foremost cause of cancer related deaths throughout the world. Approximately $75-85 \%$ of all lung cancers belong to non-small-cell lung cancer (NSCLC), a belligerent tumor type with a 5 year survival rate of only $16 \%$ that has improved little over the last 35 years (Jemal et al., 2010). Lung cancer causes approximately 1.4 million deaths annually, as reported in 2008 (Ferlay et al., 2010). Resistance to chemotherapy is one of the main obstacles for the treatment of lung cancers. Platinum-based drugs are widely used to treat patients with NSCLC in clinics. Nevertheless, drugresistance frequently develops in these patients. For example, approximately $70 \%$ of NSCLC patients with advanced unresectable or prevalent incurable metastasis are candidates for neoadjuvant or palliative chemo -therapy. However, approximately two-thirds of these patients do not respond to conventional chemotherapy (Ferreira et al., 1999). Consequently, there is an urgent need to search for new chemotherapeutic agents with enhanced activity and less chances of getting resistance against lung cancer. In the past few decades, a great number of plant-derived bioactive compounds have been isolated that are now extensively used to treat different malignant cancers. The important goal of cancer drug discovery is to develop therapeutic agents that are effective, safe, and affordable. An integrative approach for managing a patient with lung cancer should target the multiple biochemical and physiologic pathways that support tumor development and minimize normal-tissue toxicity.

In the current study, we have reported the anticancer and apoptosis inducing properties of a triazolyl analogue of alantolactone.

\section{Materials and Methods \\ Chemicals and other reagents}

Alantolactone, diazoethane and triethyl ammine was 
purchased from Sigma Chemical Company (St. Louis. Co). Deionized water was used in all experiments. Dulbecco's modified Eagle's medium, fetal bovine serum (FBS), penicillin-streptomycin were obtained from Hangzhou Sijiqing Biological Products Co., Ltd, China. MTT kit was obtained from Roche (USA). Annexin V-FITC-propidium iodide apoptosis detection kit was purchased from (Beyotime Institute of Biotechnology, Shanghai, China). Hoechst 33258 was purchased from Beyotime, China). All other chemicals and solvents used were of the highest purity grade. Cell culture plastic ware was bought from BD Falcon (USA).

General procedure for synthesis of alantolactone pyrazoline analogue from alantolactone (1) and diazomethane (Scheme 1)

To the solution of alantolactone (1, $5 \mathrm{~g}$ ) in ether containing 5-10 drops of triethylamine was added in excess etheral solution of diazoethane. It was kept overnight. After completion of reaction as checked by TLC, the solvent was evaporated to afford crystalline compound $(2,4.2 \mathrm{~g})$ identified as pyrazoline analogue of alantolactone $\left(118.2^{\circ} \mathrm{C}\right)$. All the melting points are uncorrected and were determined in open capillaries, on a BüchiB-542 melting point apparatus. The purity of the compounds was checked by TLC and RP-HPLC. IR spectra were measured in $\mathrm{CHCl}_{3}$ solution on Perkin Elmer, Model RX-1 FTIR spectrophotometer. All NMR spectra were recorded on Bruker DPX 400 instrument using $\mathrm{CDCl}_{3}$ as the solvent with TMS as internal standard. Mass spectra were recorded on ESI-esquire 3000 Bruker Daltonics instrument.

\section{Cell line and cell viability evaluation by MTT assay}

Human non-small cell lung cancer cell line (NCI-H460) was purchased from Shanghai Institute of Biochemistry and Cell Biology (Shanghai, China) and was kept at $37^{\circ}$ $\mathrm{C}$ in a humidified atmosphere containing $5 \% \mathrm{CO}_{2}$. The cells were cultured in Dulbecco's modified Eagle's media supplemented with $10 \%$ fetal bovine serum and $100 \mathrm{U} / \mathrm{mL}$ penicillin and $100 \mu \mathrm{g} / \mathrm{mL}$ streptomycin. Inhibition of cell proliferation by alantolactone pyrazoline analogue was evaluated by the MTT assay. Briefly, cells were plated in 96-well culture plates $(1 \times$ $10^{5}$ cells/well). After 24 hours incubation, cells were treated with alan-tolactone pyrazoline analogue $(0,5$, $25,75,100$ and $120 \mu \mathrm{M}$, eight wells per concentration) for 12,36 and 72 hours, MTT solution $(5 \mathrm{mg} / \mathrm{mL})$ was then added to each well. After 4 hours incubation, the formazan precipitate was dissolved in $100 \mu \mathrm{L}$ dimethyl sulfoxide, and then the absorbance was measured in an ELISA reader (Thermo Molecular Devices Co., Union City, USA) at $570 \mathrm{~nm}$. The cell viability ratio was calculated by the following formula:

Inhibitory ratio $(\%)=(\mathrm{OD}$ control $-\mathrm{OD}$ treated $) / \mathrm{OD}$ control $\times 100 \%$.

Cytotoxicity was expressed as the concentration of alantolactone pyrazoline analogue inhibiting cell growth by $50 \%$ ( $\mathrm{IC}_{50}$ value).

\section{Colony formation assay}

NCI-H460 cells were suspended in $1 \mathrm{ml}$ of DMEM containing $0.5 \%$ agarose (Amresco, USA) and 10\% FBS, and plated on a bottom layer con-taining $0.8 \%$ agarose and $10 \%$ FBS in 6-well plate in triplicate. After 1 week, plates were stained with $0.5 \%$ gentian violet and the colonies were counted under light microscope (Ma et al., 2011).

\section{Invasion assay}

This assay was done with a 24-well plate. Matrigel (BD) coating was carried out on a polyvinyl-pyrrolidone-free polycarbonate filter (5 $\mathrm{mm}$ pore size). The lower chamber was filled with medium containing $10 \%$ FBS. NCI-H460 cells $\left(1 \times 10^{5}\right.$ cells/well) were preincubated with alantolactone pyrazoline analogue for $30 \mathrm{~min}$ at room temperature and the cell medium containing alantolactone pyrazoline analogue was seeded onto the upper chamber wells. Following incubation for 48 hours, the filter was fixed and stained with $2.5 \%$ ethanol containing $0.5 \%$ crystal violet for $15 \mathrm{~min}$. The stained cells were counted under light microscope.

\section{Morphological study of apoptosis using Hoechst 33258 staining}

Human NSCLC cells (NCI-H460 cells) (1x $10^{5}$ cells/ dish) were plated in 6-cm dish and then treated with or without alantolactone pyrazoline analogue $(0,25,75$ and $120 \mu \mathrm{M})$. After 48 hours incubation, the harvested cells were washed with PBS and fixed with 1.5\% glutaraldehyde for $30 \mathrm{~min}$. After this the cells were stained with Hoechst 33258 for $15 \mathrm{~min}$. The cells were again washed with PBS and then observed by fluorescence microscope (Olympus, Olympus Optical Co., LTD, Tokyo, Japan) using UV filter at $40 \mathrm{X}$ magnification.

Effect of alantolactone pyrazoline analogue on cell cycle phase distribution

Briefly, NSCLC cells (NCI-H460 cells) $\left(1 \times 10^{6}\right.$ cells $\left./ \mathrm{mL}\right)$ were seeded into each well of 6-well plates and incubated for 24 hours for cell attachment and recovery. The cells were treated with different concentrations $(0$, 25, 75 and $120 \mu \mathrm{M}$ ) of alantolactone pyrazoline analogue for 48 hours. After incubation for 24 hours, the cells were harvested and fixed with ice-cold $70 \%$ ethanol $(2 \mathrm{~mL})$ at $-20^{\circ} \mathrm{C}$ for 1 hour. Before analysis, the cells were washed with cold PBS and re-suspended in $400 \mu \mathrm{L}$ of PBS, $50 \mu \mathrm{L}$ PI and $50 \mu \mathrm{L}$ RNase A. The DNA contents were recorded by a FACS Calibur flow 


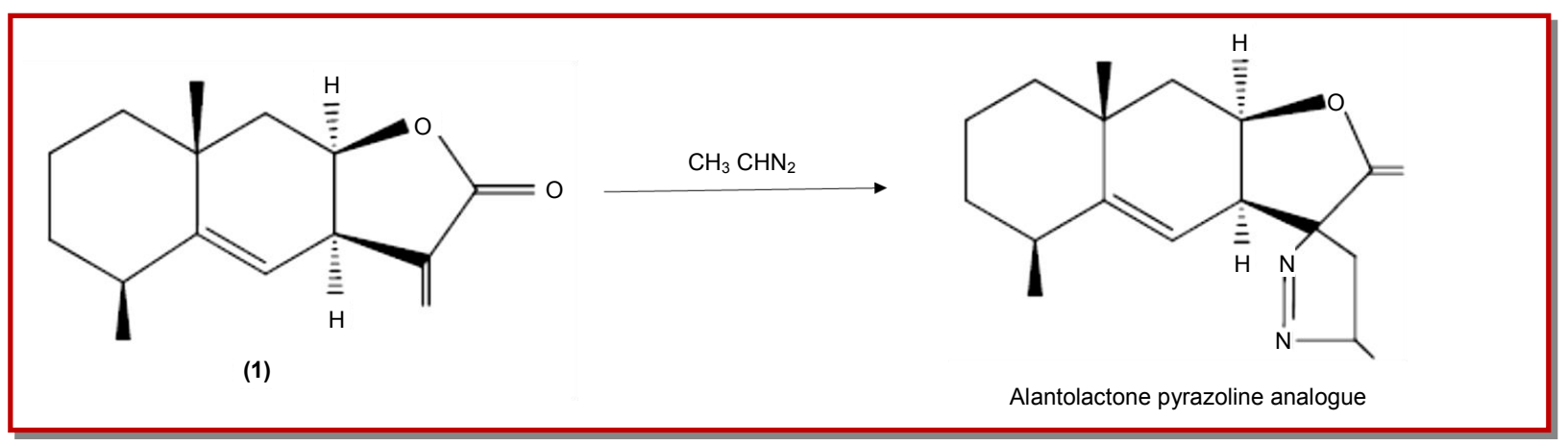

Scheme 1: General procedure for synthesis of alantolactone pyrazoline analogue

cytometer (Becton, Dickinson and Company, Franklin Lakes, NJ, USA) equipped with Cell Quest software.

\section{Statistical Analysis}

All data were derived from at least three independent experiments. The results were expressed as the mean \pm SD. Differences between groups were analyzed using the Student's t-test. $\mathrm{p}<0.05$ was considered statistically significant.

\section{Results}

As can be seen from the Figure 1, the compound exhibited dose-dependent as well as time-dependent antiproliferative effects against the cell line. The results are encouraging since this cell line is highly resistant to most anticancer drugs and such APA can be developed as a possible chemotherapeutic agent against non-small cell carcinoma.
APA also inhibited the clonogenic activity of the NCIH460 cancer cells by dropping the number of cancer colony forming cells. Clonogenic reduction also followed the concentration dependence on APA (Figure 2). The results indicate that APA has the tendency to inhibit both cell proliferation (anchorage-dependent) and colony formation (anchorage-independent) growth of NSCLC cells in vitro. APA dose of $120 \mu \mathrm{M}$ could reduce the number of cancer forming colonies by $88.2 \%$.

Lung cancer is very lethal because it is extremely invasive specifically in later stages. In this experiment related to APA, we demonstrated whether APA could inhibit the invasive behavior of NSCLC (NCI-H460) cells. The invasive assay was designed using NCI-H460 cells using Matrigel-coated 24-well microchemotaxis chambers in the presence of APA at various concentrations $(0,5,25,75$ and $120 \mu \mathrm{M})$. As can be seen in Figure 3, APA considerably inhibited the invasion of NCI-H460 cells in a dose-dependent manner.

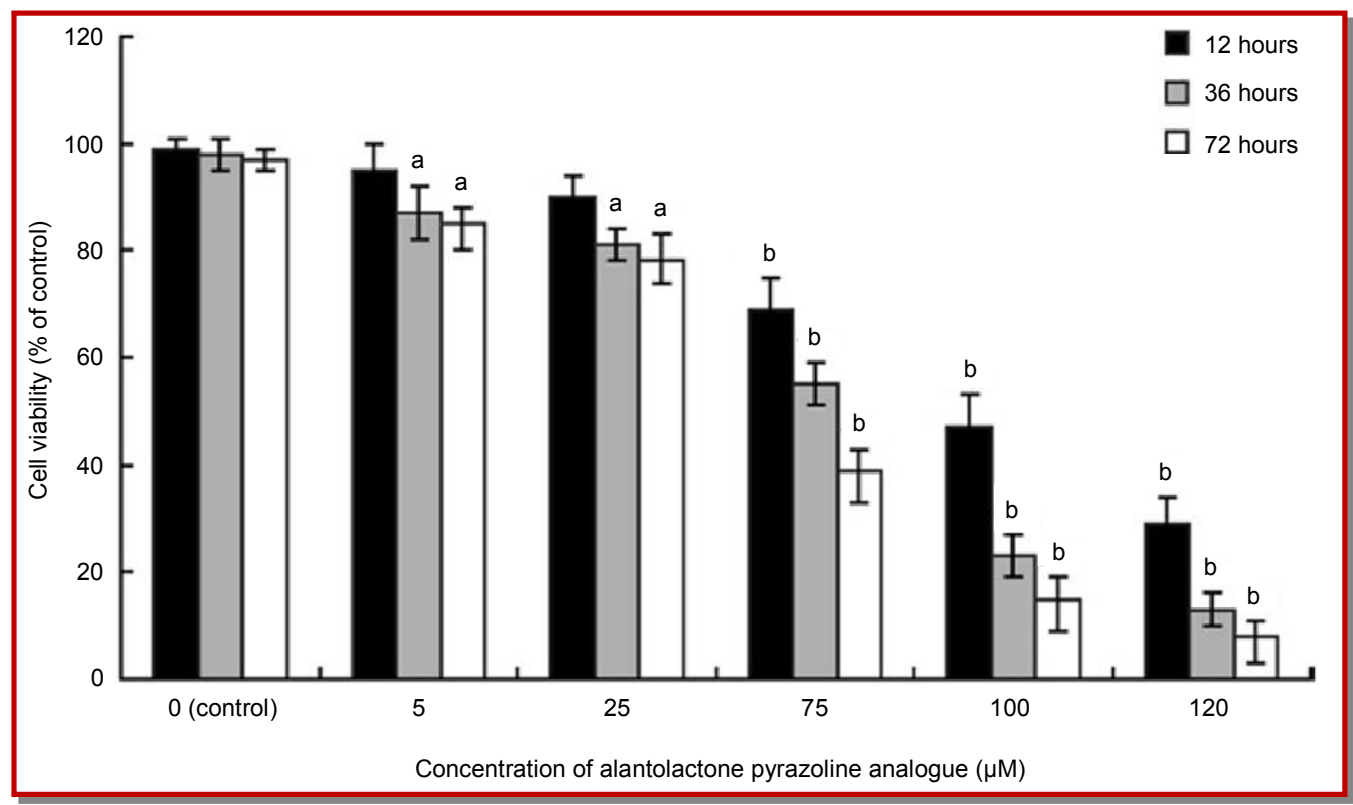

Figure 1: Effect of alantolactone pyrazoline analogue (APA) on cytotoxicity of NSCLC cells. The cells were treated with APA at the indicated concentration for 12, 36, and 72 hours. Cell viability was assessed by MTT assay. The data are expressed as percentage of control and each value represents mean $\pm S D$ of three independent experiments. ${ }^{a} p<0.05,{ }^{b} p<0.01$ versus control 


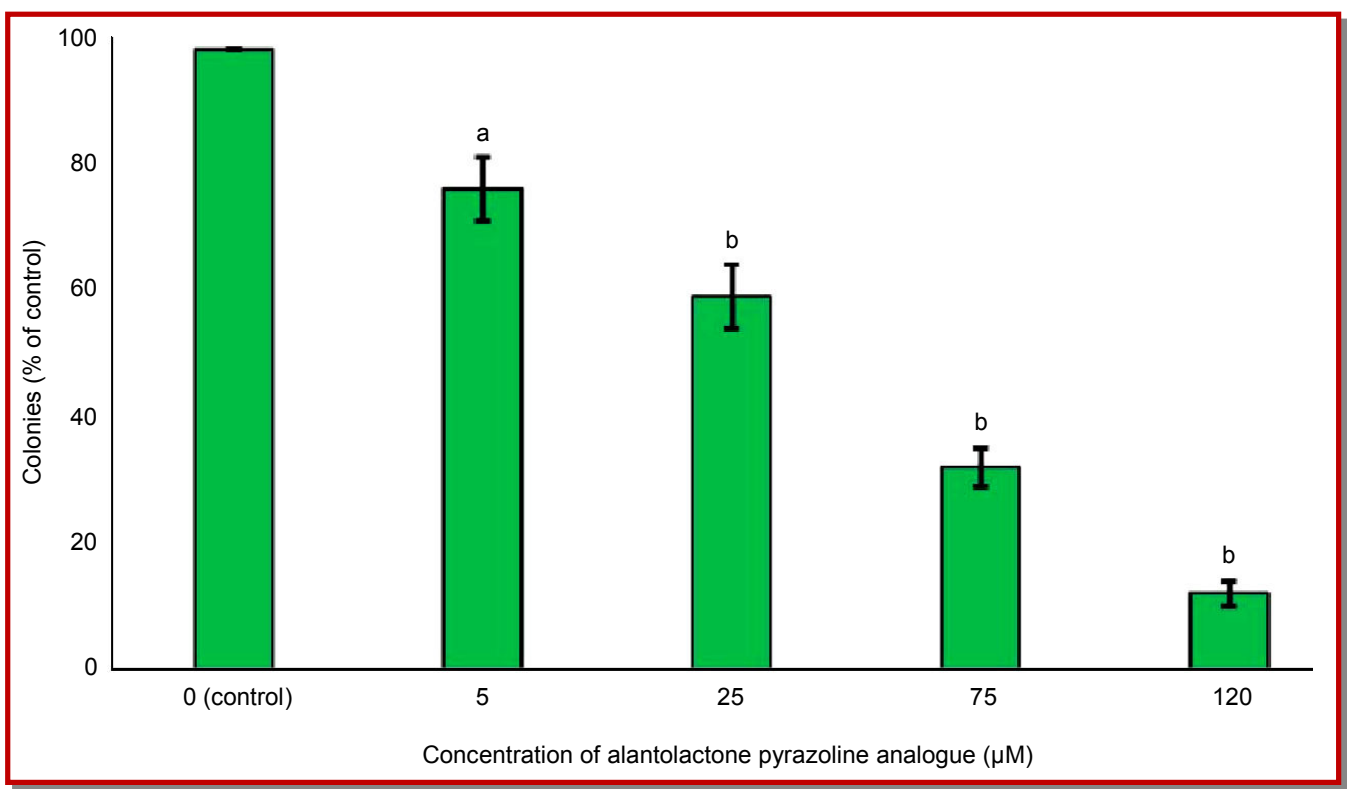

Figure 2: Inhibitory effect of alantolactone pyrazoline analogue (APA) on human non-small cell lung cancer cells. The colony formation assay of NSCLC cells treated with APA at indicated doses. Data are shown as the mean \pm SD of three independent experiments by analysis of Student's $t$ test. ap $<0.05$, b $p<0.01$, vs $0 \mu \mathrm{M}$ (control)

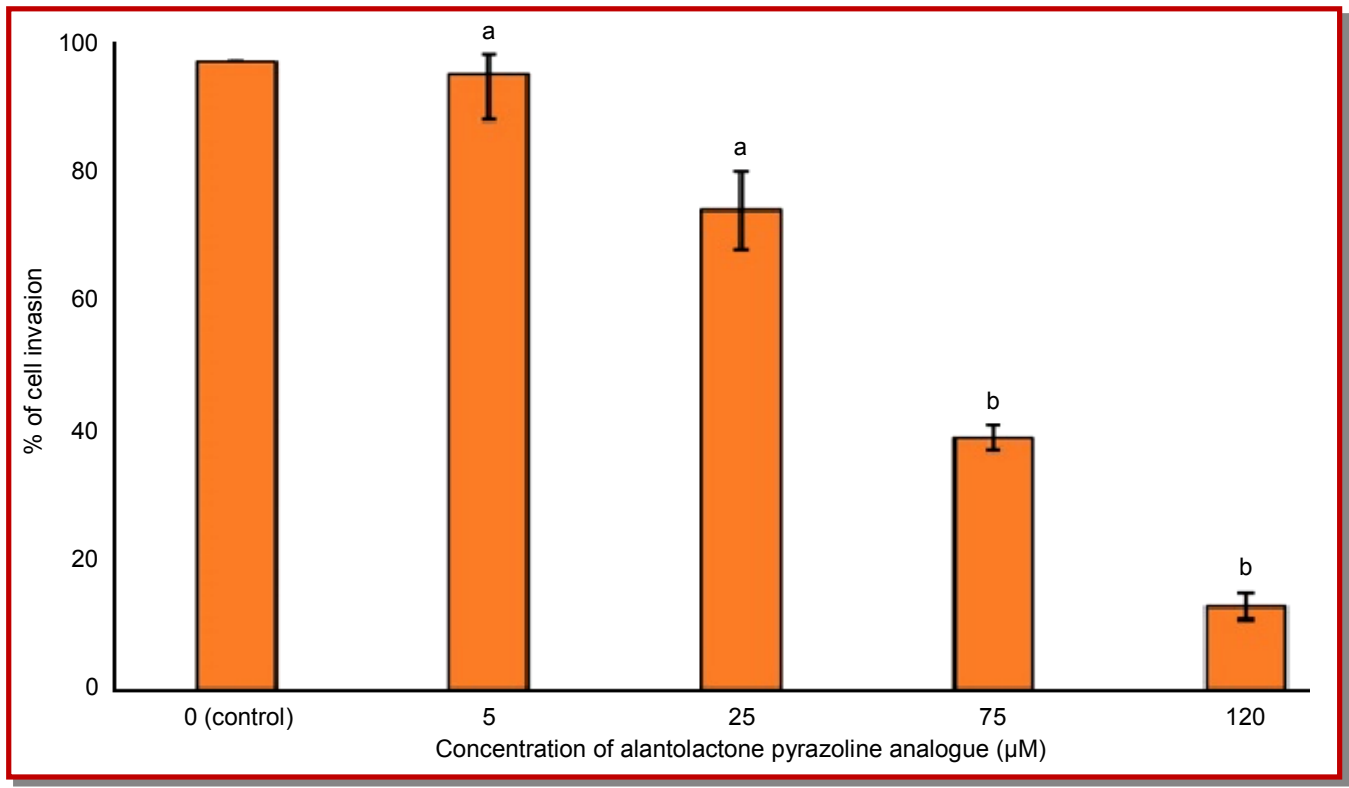

Figure 3: Graphical representation of the alantolactone pyrazoline analogue-induced reduction in the invasion of non-small cell lung cancer cells. Data are shown as the mean \pm SD of three independent experiments by analysis of Student's $t$-test. ap $<0.05$; ${ }^{b} \mathrm{p}<0.01$ vs $0 \mu \mathrm{M}$ (control)

We further evaluated the effect of APA on NCI-H460 cellular morphology and apoptosis using fluorescence microscopy and Hoechst 33258 staining. NCI-H460 cells were subjected to APA treatment using different concentrations $(0,25,75$ and $120 \mu \mathrm{M})$. APA induced significant nuclear morphological changes including chromatin condensation and DNA fragmentation which can easily be seen in Figure 4. These nuclear morphological changes are hallmark of apoptosis. The bright blue fluorescence indicates chromatin condensation and DNA fragmentation. Figure 4A represents untreated cells which showed normal nuclear morphology. Figure $4 \mathrm{~B}-\mathrm{D}$ represent 25,75 and $120 \mu \mathrm{M}$ doses of APA respectively. These effects of APA increased with increasing concentration of APA. The extent of apoptosis is shown in Figure 5. The compound affected both early and late apoptosis of the cells. 

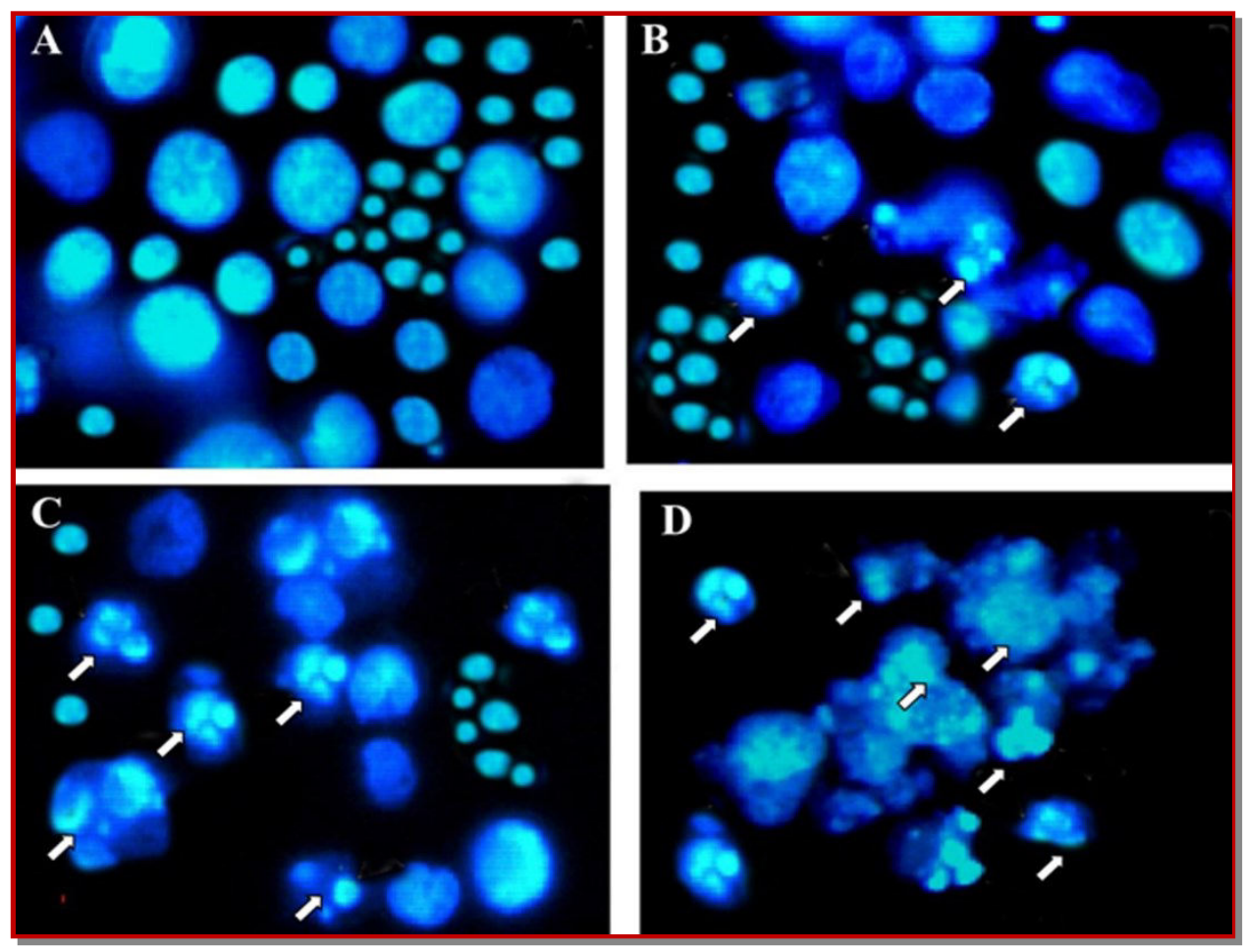

Figure 4: Effect of alantolactone pyrazoline analogue on nuclear morphology (chromatin condensation) in NSCLC cells. The cells were treated with $0 \mu \mathrm{M}$ (A, untreated control), $25 \mu \mathrm{M}$ (B), $75 \mu \mathrm{M}(\mathrm{C})$ and $120 \mu \mathrm{M}$ (D) respectively for $48 \mathrm{~h}$ and stained with Hoechst 33258. The nuclear morphology was observed by fluorescent microscope (magnification 500×). White arrows represent nuclear chromatin condensation and apoptotic body formation

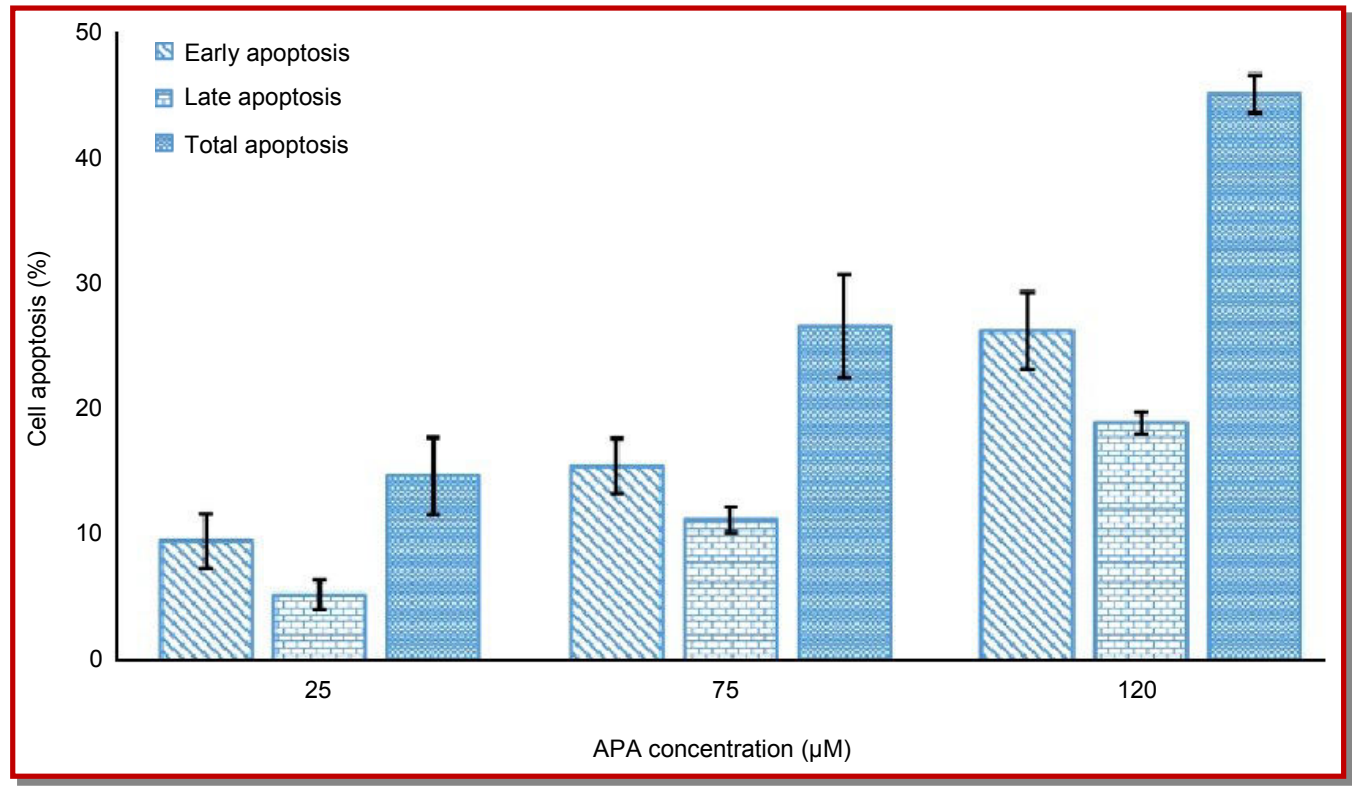

Figure 5: Percentages of cells in early and late apoptosis at each APA concentration. Results are presented as the mean of three similar experiments \pm standard deviation

In the current study, we also assessed the effect of APA on cell cycle phase distribution in NCI-H460 lung cancer cells using flow cytometry and propidium iodide staining. APA induced potent cell cycle arrest in G0/G1 (apoptotic phase) phase of the cell cycle and this effect was found to follow the concentration of APA (Figure 6A-D). The percentage of apoptotic cells (G0/G1 cells) increased from $1.3 \%$ in control (A), to $5.6 \%$ (B), $15.6 \%$ (C) and $46.7 \%$ (D) in cells treated with 25, 75 and 120 $\mu \mathrm{M}$ dose of APA respectively. 


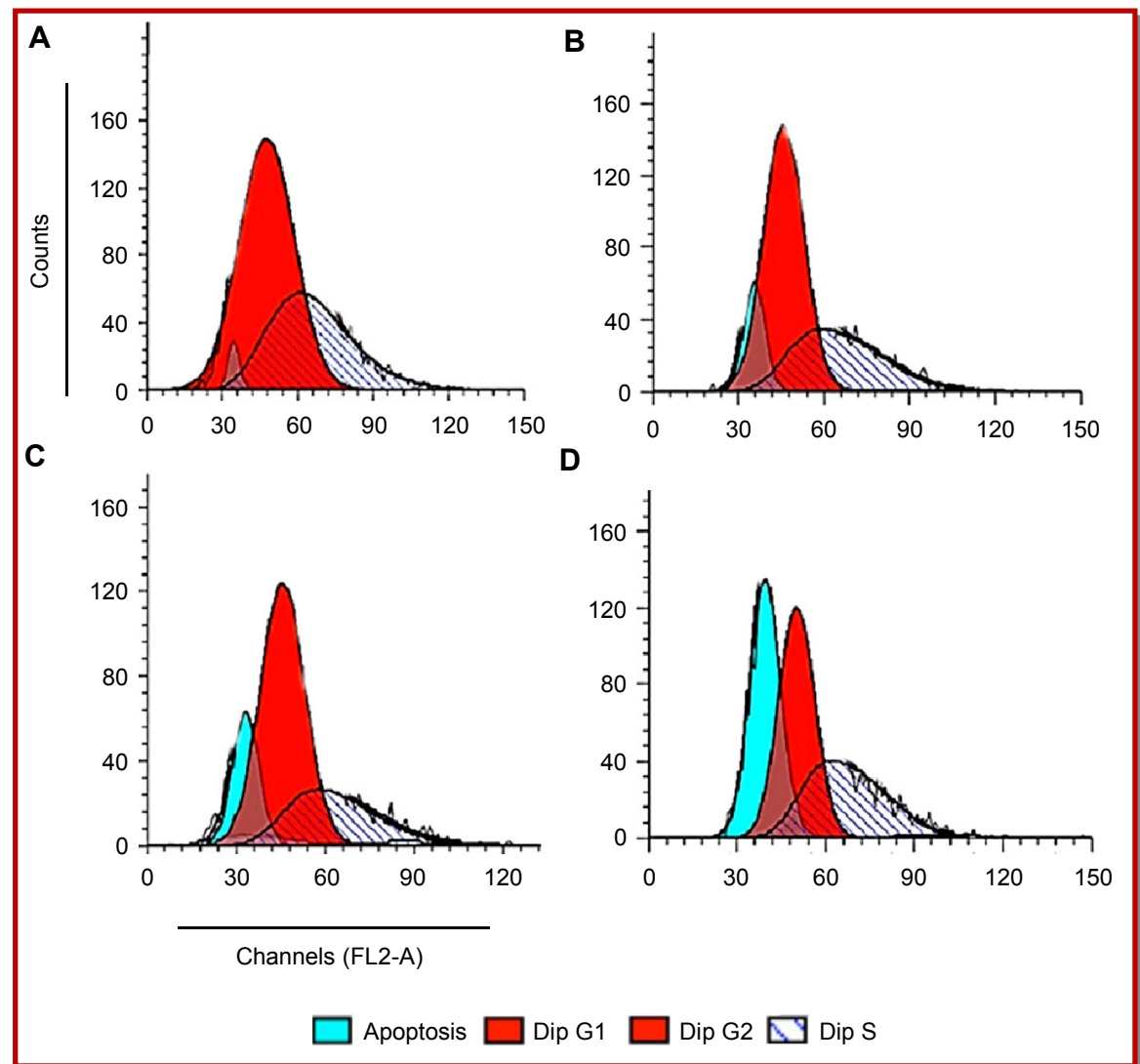

Figure 6: Effect of alantolactone pyrazoline analogue (APA) on cell cycle in NSCLC cells. The cells were subjected to $0 \mu \mathrm{M}$ (untreated, A), $25 \mu \mathrm{M}$ (B), $75 \mu \mathrm{M}$ (C) and $120 \mu \mathrm{M}$ (D) of APA. The apoptotic cells increased from A-D, with an increase in APA dose. The cell cycle distribution was determined by a flow cytometric analysis of the DNA content after staining with propidium iodide

\section{Discussion}

Alantolactone is a sesquiterpene lactone usually isolated from the inula plant species especially from the roots of Inula racemosa commonly known as Pushkarmoola, a plant of wide medicinal importance which belongs to family Asteraceae and has its origin in temperate and Alpine Western Himalayas (Kalsi et al., 1995). Previous published reports claim that alantolactone selectively suppresses STST3 activation and exhibits anticancer activity in breast cancer (MDA-MB231) cells. This sesquiterpene lactone has also been reported to induce apoptosis in HepG2 cells through GSH depletion and mitochondrial dysfunction (Jaemoo et al., 2015; Khan et al., 2013). Alantolactone has also been reported to induce apoptosis in glioblastoma cells (Khan et al., 2012). However, no work has been reported so far on the anticancer and apoptotic effects of pyrazoline analogue of alantolactone against nonsmall cell lung carcinoma.

In the present research work, we evaluated the inhibitory effect of alantolactone pyrazoline analogue in NCI-H460 lung cancer migration. Percentage of wound closure was determined by the difference in area covered by migrated cells in control versus treated with alantolactone pyrazoline analogue for 24 hours. The compound showed potent inhibitory effects against cell migration. Apoptosis induction in cancer cells is also seen as a potent mechanism through which a wide variety of natural phytochemicals suppress cancer growth. The biological mechanisms of antitumor drugs include the inhibition of proliferation, growth arrest in the cell cycle, increased apoptosis and the modulation of signal transduction pathways. Numerous natural phytochemicals have been reported to inhibit the growth of cancer cells through disruption of cell cycle progression (Chen and Kong, 2005; Aggarwal et al., 2004; Hartwell and Kastan, 1994). Alantolactone pyrazoline analogue was able to induce potent apoptosis, both early and late apoptosis. The compound also induced G0/G1 cell cycle arrest which may explain its tendency to induce apoptosis.

In conclusion, alantolactone pyrazoline analogue shows potent anticancer effects by inducing apoptosis, inhibiting cell invasion and cell cycle arrest. 


\section{References}

Aggarwal BB, Takada Y, Oommen OV. From chemoprevention to chemotherapy: Common targets and common goals. Expert Opin Investig Drugs. 2004; 13: 1327-38.

Chen C, Kong AN. Dietary cancer-chemopreventive compounds: From signaling and gene expression to pharmacological effects. Trends Pharmacol Sci. 2005; 26: 318-26.

Chun J, Li RJ, Cheng MS, Kim YS. Alantolactone selectively suppresses STAT3 activation and exhibits potent anticancer activity in MDA-MB-231 cells. Cancer Lett. 2015; 357: 393403.

Ferlay J, Shin HR, Bray F, Forman D, Mathers C, Parkin DM. Estimates of worldwide burden of cancer in 2008: GLOBOCAN 2008. Int J Cancer. 2010; 127: 2893-917.

Ferreira CG, Tolis C, Giaccone G. p53 and chemosensitivity. Ann Oncol. 1999; 10: 1011-21.

Hartwell LH, Kastan MB. Cell cycle control and cancer. Science 1994; 266: 1821-28.

Jemal A, Siegel R, Xu J, Ward E. Cancer statistics, 2010. CA
Cancer J Clin. 2010; 60: 277-300.

Kalsi PS, Kumara S, Jawanda GS, Chhabra BR. Guaianolides from Saussurea lappa. Phytochemistry 1995; 40: 1713-15.

Khan M, Li T, Ahmad Khan MK, Rasul A, Nawaz F, Sun M, Zheng Y, Ma T. Alantolactone induces apoptosis in HepG2 cells through GSH depletion, inhibition of STAT3 activation, and mitochondrial dysfunction. Biomed Res Int. 2013; 2013: 719858 .

Khan M, Yi F, Rasul A, Li T, Wang N, Gao H, Gao R, Ma T. Alantolactone induces apoptosis in glioblastoma cells via GSH depletion, ROS generation, and mitochondrial dysfunction. IUBMB Life. 2012; 64: 783-94.

Ma L, Wen ZS, Liu Z, Hu Z, Ma J, Chen XQ, Liu YQ, Pu JX, Xiao WL, Sun HD, Zhou GB. Overexpression and small molecule-triggered down-regulation of CIP2A in lung cancer. PLoS One. 2011; 6: e20159.

Peng C, Zhou K, An S, Yang J. The effect of CCL19/CCR7 on the proliferation and migration of cell in prostate cancer. Tumour Biol. 2015; 36: 329-35. 